\title{
THE
}

$12-5-2002$

\section{Topological Phase Transitions in Periodically Modulated Quantized Metal Films}

\author{
A. E. Meyerovich \\ University of Rhode Island, sfo101@uri.edu \\ Dongmin Chen
}

Follow this and additional works at: https://digitalcommons.uri.edu/phys_facpubs

Terms of Use

All rights reserved under copyright.

\section{Citation/Publisher Attribution}

Meyerovich, A. E., \& Chen, D. (2002). Topological Phase Transitions in Periodically Modulated Quantized Metal Films. Phys. Rev. B, 66(23), 235306. doi: 10.1103/PhysRevB.66.235306

Available at: http://dx.doi.org/10.1103/PhysRevB.66.235306

This Article is brought to you for free and open access by the Physics at DigitalCommons@URI. It has been accepted for inclusion in Physics Faculty Publications by an authorized administrator of DigitalCommons@URI. For more information, please contact digitalcommons-group@uri.edu. 


\title{
Topological phase transitions in periodically modulated quantized metal films
}

\author{
Alexander E. Meyerovich ${ }^{1}$ and Dongmin Chen $^{2}$ \\ ${ }^{1}$ Department of Physics, University of Rhode Island, Kingston, Rhode Island 02881 \\ ${ }^{2}$ The Rowland Institute at Harvard, Harvard University, Cambridge, Massachusetts 02142
}

(Received 30 August 2002; published 5 December 2002)

\begin{abstract}
Electronic topological phase transitions (ETT) are predicted for any quantized metal films with a periodically modulated boundary commensurate with the bulk lattice. While the thickness of the film at which ETT occurs is determined almost exclusively by the period of the boundary modulation, the value of the band gaps and the amplitude of the singularities are proportional to the amplitude of the corrugation. The gaps in the energy spectrum lead to a strong anisotropy of conductivity. These novel quantum effects can be detected in measurements of the density of states and conductivity as a function of the film thickness. Experiments that could lead to these observations are proposed.
\end{abstract}

DOI: 10.1103/PhysRevB.66.235306

Boundary condition is an essential part of a complete description of a quantum system. Due to the large surface-tovolume ratio, boundaries are expected to play a much greater role in determining the overall properties in a nanostructure than in a bulk material. For example, recent reports have shown that scanning tunneling microscopy (STM), a wellknown near-field technique, reveals images and electron energy spectra that are more strongly correlated to the buried interfacial lattices under a thin lead $(\mathrm{Pb})$ film supported on silicon substrates than to the surface immediately beneath the STM tip. ${ }^{1}$ These surprising observations clearly indicate that a small lateral variation along the boundary can have a significant long-range effect in a semiballistic electron system. A more realistic description of a nanoscale quantized system, therefore, must go beyond the commonly adopted "perfect geometric" boundary conditions to include boundary corrugations. Indeed, theoretical investigations have shown that random surface roughness of a thin metal film can dominate incoherent scattering and relaxation, and can lead to anomalous quantum size effect such as large oscillatory dependence of the in-plane conductivity on the film thickness. ${ }^{2}$

In this paper, we show that when the inhomogeneities of the boundary are regular as shown in Fig. 1(a), electronic topological phase transition (ETT; see Ref. 3 and references therein) can occur in any quantized metal film. While the positions of ETT's are determined almost exclusively by the period of the boundary corrugation, the value of the band gaps and the amplitude of the singularities are proportional to the amplitude of the corrugation. The gaps in the energy spectrum lead to a strong anisotropy of conductivity. These novel quantum effects can be observed in measurements of the density of states or of the lateral conductivity of the film as a function of the film thickness.

As it will be clear from our approach and discussion below, the actual implications of our prediction are much broader than ETT in metal films. Similar changes in (quasi)particle spectra should occur as a result of the periodic modulation of surfaces of any films or ultrathin channels.

Recent technological advances have offered a number of possible ways for engineering such ultrathin crystalline film that has a corrugated boundary with a period of just few times of the bulk lattice and a corrugation amplitude of a few atom heights. It is by now well established that ultrathin
PACS number(s): 71.30.+h, 73.61.At, 73.63.Hs

films grown on either a different metal substrate with large band offset or a semiconductor substrate exhibit strong quantization and sharp energy spectra (long lifetime). ${ }^{4}$ There is also a collection of experimental results, which shows that adsorbates, such as oxygen, commonly induce periodic atomic corrugations on the surface of noble and transition metals in the form of one-dimensional (1D) gratings or a two-dimensional (2D) array. ${ }^{5}$ As illustrated in Fig. 1(b), combining these two effects, one can engineer a specimen that could satisfy the ETT requirements. A more interesting alternative is to fabricate thin film on a template which contains the desired periodic corrugation as shown in Fig. 1(c).

Recent works on thermal treatment of high-index surfaces of silicon or germanium have yielded a variety of such templates. ${ }^{6}$ One of the most striking results is an atomically accurate Si grating with $5.73 \mathrm{~nm}$ period made on a $\mathrm{Si}(577)$ surface. ${ }^{7}$ These structures are stable under a thin metal film of material that is suitable for low-temperature molecular epitaxy. The buried corrugated boundary makes it less ambiguous when probing the electronic density or conductivity on top of the flat surface using tools such as STM. With the improvement of the feature size, lithographic technique should enable us to engineer boundaries of arbitrary shape and symmetry in the near future. ${ }^{8}$

Below we consider quantized metal films with periodically modulated surface with the period which is larger than and commensurate with the period of the lattice. A possible theoretical approach to such a film is to perform an explicit coordinate transformation that maps a problem of particles in a film with inhomogeneous walls onto an equivalent problem with perfect flat walls and perturbed bulk Hamiltonian. ${ }^{9}$ In the case of periodic modulation, this bulk perturbation is also periodic with the same period. [An alternative to this quasiclassical technique is the ultraquantum approach of Ref. 10, which is based on the lattice Hamiltonian for a film with few atomic layers.]

A mere appearance of a new wall-driven period leads to qualitative changes. The most important one is the resizing of a Brillouin zone to the wall period instead of the smaller bulk period. The boundaries of this new, smaller Brillouin zone can intersect the Fermi surface. This, in turn, should require the reconstruction of the Fermi surface. This reconstruction usually results in the change of the topology of the 

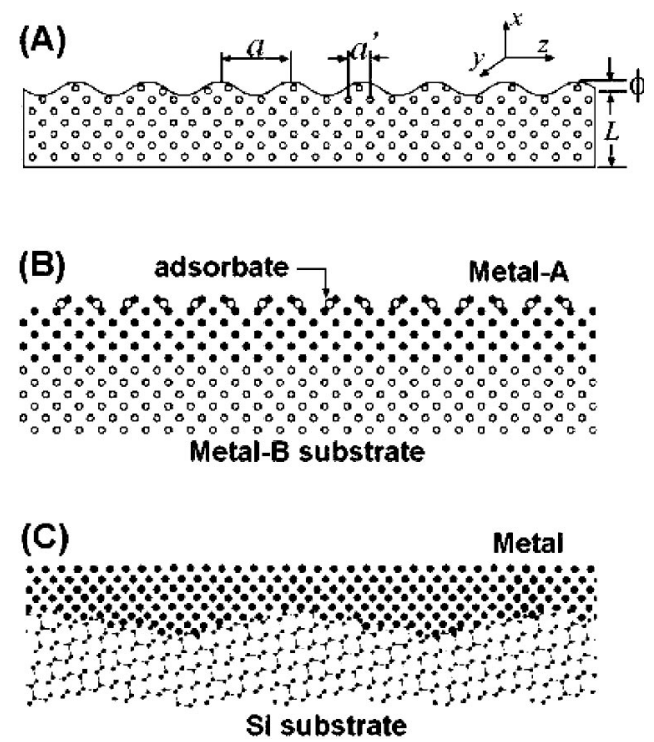

FIG. 1. (a) Schematic of an ultrathin film with a periodically corrugated boundary on one side; two ways to make such films are shown in (b) and (c). In (b), an utlrathin film of metal $A$ is grown on the metal $\mathrm{B}$, the latter having a large band offset from the former. Adsorbate on the surface of metal $A$ induces periodic corrugation. In (c), a periodically corrugated surface of a high-index silicon is used as a template for growing metal $A$ on top. The corrugated boundary is buried and protected under the metal film.

Fermi surface such as formation of new loops, formation or disruption of the necks, etc. The reconstruction can be also accompanied by the change in the hierarchy of the band gaps, and can even lead to a metal-dielectric transition if the new cell contains an even number of electrons.

The most significant modulation-driven changes in the energy spectra occur, as always, near the cell boundaries and other degeneracy (Bragg) points near which the changes are linear in the perturbation and are much larger than the quadratic corrections elsewhere. In metal films, this is crucial when the Fermi surface is close to the edges of the Brillouin

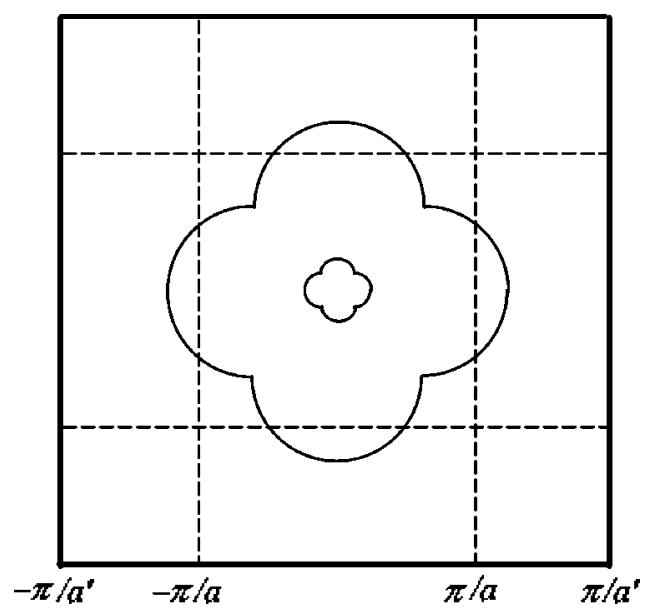

FIG. 2. Schematic drawing of the Fermi surface for quantized film when the Fermi energy and the film thickness allow only four quantized states $j= \pm 1, \pm 2$. Solid borders-bulk Brillouin zone $\left(-\pi a^{\prime}, \pi a^{\prime}\right)$; dashed lines—surface-driven Brillouin zone $(-\pi a, \pi a)$.

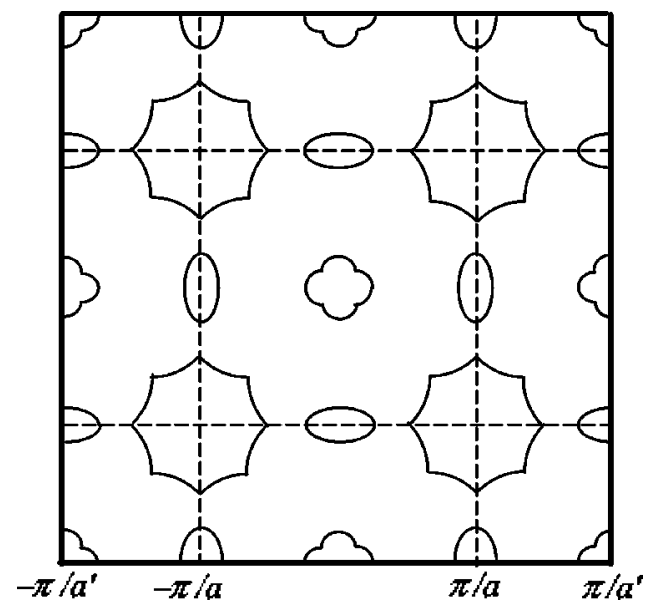

FIG. 3. Schematic drawing of the reconstructed Fermi surface obtained from Fig. 2 after the periodic modulation of the film surface. Solid borders-bulk "old" Brillouin zone $\left(-\pi a^{\prime}, \pi a^{\prime}\right)$; dashed lines-new surface-driven Brillouin zone $(-\pi a, \pi a)$. The inner Fermi curve does not change while the outer one undergoes an obvious topological transition. The separation between parts of the curve on the zone boundary is determined by the energy gaps $\delta E$.

zone. The significance of the changes depends on comparison of the surface-driven perturbation to the scatteringdriven broadening of the spectra and the Fermi surface. Below we analyze the interplay between the quantum size effect (QSE) and the electronic topological phase transition (ETT; see Ref. 3 and references therein).

Qualitatively, the predicted effects are transparent. QSE in ultrathin films corresponds to the quantization of motion in the direction perpendicular to the film, $p_{x}=\pi j / L$ (here and below, $\hbar=1)$. The Fermi surface of such a film is a set of $2 \mathrm{D}$ curves $\epsilon\left(\pi j / L, \mathbf{q}_{j}\right)=\epsilon_{F}$, which are produced by cutting the bulk Fermi surface $\epsilon\left(\mathbf{p}_{F}\right)=\epsilon_{F}$ by the planes $p_{x}=\pi j / L\left(\mathbf{q}_{j}\right.$ is the Fermi momentum in the $y-z$ plane for each $j$ ). This type of the Fermi surface is presented schematically in Fig. 2 when the film parameters allow only four values of $p_{x}=$ $\pm \pi / L, \pm 2 \pi / L$; and the Fermi surface consists of four curves.

The sizes of the curves $\mathbf{q}_{j}$ and their number strongly depend on the film thickness $L$. With increasing $L$, the dissecting planes $p_{x}=\pi j / L$ converge towards the plane $p_{x}=0$, making the Fermi curves in Fig. 2 to expand. Also, at certain values of $L$, new Fermi curves are nucleated in the center of the zone, leading to the changes in the topology of the Fermi surface and creating singularities in the density of states, conductivity, ultraviolet spectra, etc. These singularities are the well-known standard features of the QSE. Periodic modulation of the film surface results in a quite different series of the topological transformations.

Periodic modulation leads to the drastic decrease in the size of the Brillouin zone (dashed lines in Figs. 2 and 3). This does not affect much the smaller Fermi curves that fit inside the "new" Brillouin zone. However, since the new zone boundaries intersect the bigger Fermi curves, the topology of these Fermi curves undergoes dramatic changes. The corresponding reconstruction of the Fermi curves from Fig. 2 is presented schematically in Fig. 3. This ETT occurs at the 
value of the film thickness $L_{1}$, at which the Fermi curve first touches the Brillouin-zone boundary. With a further increase in $L$, the inner (previously unaffected), Fermi curves expand and, at a certain value $L_{2}$, the second one touches the new zone boundary and undergoes the ETT, then the next curve, etc. In contrast to the usual ETT in metals, which can be observed only if the Fermi surface is accidentally very close to the zone boundary, ${ }^{3}$ the above series of ETT's is inevitable, as a function of thickness, in any quantized metal film with periodically modulated surfaces. This ETT is best observed as a function of $L$ in STM or conductivity experiments in films with a modulated buried interface.

The following simplified description assumes that the initial Fermi surface is ellipsoidal, as in $\mathrm{Pb}$, with two effective masses, $\boldsymbol{\epsilon}_{F}=p_{x}^{2} / 2 m_{\perp}+q^{2} / 2 m_{\|}$. As a result of the size quantization in the ultrathin film, $p_{x}=\pi j / L$, the Fermi surface becomes a set of circles,

$$
q_{j}^{2}(L)=2 m_{\|} \boldsymbol{\epsilon}_{F}-\frac{m_{\|}}{m_{\perp}}\left(\frac{\pi j}{L}\right)^{2} .
$$

Experiments are performed either at constant $\epsilon_{F}$ or at constant 2D density of electrons, $n_{2}$. The link between $\epsilon_{F}$ and $n_{2}$ is

$$
n_{2}=\frac{S}{2 \pi}\left[2 m_{\|} \epsilon_{F}-\frac{m_{\|}}{6 m_{\perp}}\left(\frac{\pi}{L}\right)^{2}[(S / 2)+1](S+1)\right],
$$

where $S(L)=2 \operatorname{Int}\left[(L / \pi) \sqrt{2 m_{\|} \epsilon_{F}}\right]$ is the number of the Fermi curves.

For simplicity, we assume that the film of the average thickness $L$ has one flat wall, $x=L / 2$, and one buried corrugated wall, $x=-L / 2+\Phi(y, z)$, where $\Phi(y, z)$ is a periodic function with one harmonic in each direction:

$$
\Phi(y, z)=2 \Phi_{0}\left[\cos \frac{2 \pi y}{a}+\cos \frac{2 \pi z}{a}\right] .
$$

The derivation below can be easily extended to the situation where both walls are corrugated with multiple harmonics and a nonorthogonal basis. The new Brillouin zone is a square, $-\pi / a \leqslant q_{y}, q_{z} \leqslant \pi / a$. ETT occurs when the Fermi circles $q_{j}$ touch the new zone boundaries, $q_{j}=\pi / a$, i.e., when the film thickness $L=L_{j}$,

$$
L_{j}=\pi j\left(\frac{m_{\|}}{m_{\perp}}\right)^{1 / 2}\left[2 m_{\|} \epsilon_{F}-\frac{\pi^{2}}{a^{2}}\right]^{-1 / 2} .
$$

There are also auxiliary, less pronounced singularities at $q_{j}$ $=n \pi / a$,

$$
L_{j n}=\pi j\left(\frac{m_{\|}}{m_{\perp}}\right)^{1 / 2}\left[2 m_{\|} \epsilon_{F}-n^{2} \frac{\pi^{2}}{a^{2}}\right]^{-1 / 2} .
$$

These positions of the ETT do not depend on the amplitude of the surface corrugation $\Phi_{0}$ and, for good metals, are not affected much by the lattice potential. The amplitude $\Phi_{0}$ determines the band gaps $\delta E$, reconstruction of the Fermi surface, and the magnitude of the experimental signs of ETT.

Our approach to corrugated films is based on the coordinate transformation

$$
X=\frac{L[x-\Phi(y, z) / 2]}{L-\Phi(y, z)},
$$

$Y=y, Z=z$. This transformation makes the walls flat, $X=$ $\pm L / 2$, but changes the particle momenta, $\hat{\mathbf{p}} \rightarrow \hat{\mathbf{P}}$, and as a result, adds a periodic perturbation $\hat{V}$ to the bulk Hamiltonian $\hat{H}=\hat{p}_{x}^{2} / 2 m_{\perp}+\left(\hat{p}_{y}^{2}+\hat{p}_{z}^{2}\right) / 2 m_{\|}$,

$$
\begin{aligned}
\hat{V}= & \frac{\Phi}{m_{\perp} L} \hat{P}_{x}^{2}+\frac{1}{2 m_{\|}} \times \sum_{i=y, z}\left[\Phi_{i}^{\prime}\left(\frac{X}{L}-\frac{1}{2}\right) \hat{P}_{x} \hat{P}_{i}+\hat{P}_{i} \Phi_{i}^{\prime}\right. \\
& \left.\times\left(\frac{X}{L}-\frac{1}{2}\right) \hat{P}_{x}\right] .
\end{aligned}
$$

[Though there is another periodic perturbation that arises from the application of the transformation (6) to the bulk lattice potential, in good metals the contribution from Eq. (7) is usually stronger.] The corrections to the spectrum away from the Bragg points are quadratic in the matrix elements of $\hat{V}$,

$$
\begin{gathered}
E_{j m n}=E_{j m n}^{(0)}+\sum_{j^{\prime}, m^{\prime}, n^{\prime}} \frac{\left|V_{j^{\prime} m^{\prime} n^{\prime}}^{j m n}\right|^{2}\left(1-\delta_{j^{\prime}}^{j} \delta_{m^{\prime}}^{m} \delta_{n^{\prime}}^{n}\right)}{E_{j m n}^{(0)}(\mathbf{q})-E_{j^{\prime} m^{\prime} n^{\prime}}^{(0)}(\mathbf{q})}, \\
E_{j m n}^{(0)}(\mathbf{q})= \\
\frac{1}{2 m_{\perp}}\left(\frac{\pi j}{L}\right)^{2}+\frac{1}{2 m_{\|}}\left[\left(q_{y}-\frac{2 \pi m}{a}\right)^{2}\right. \\
\left.+\left(q_{z}-\frac{2 \pi n}{a}\right)^{2}\right] .
\end{gathered}
$$

Near the Bragg points, $E_{j m n}^{(0)}(\mathbf{q})=E_{j^{\prime} m^{\prime} n^{\prime}}^{(0)}(\mathbf{q})$, the perturbation creates gaps linear in $\hat{V}$ in the spectrum:

$$
\delta E(\mathbf{q})=2\left|V_{j m n}^{j^{\prime} m^{\prime} n^{\prime}}(\mathbf{q})\right| .
$$

Simple calculation yields

$$
\begin{aligned}
V_{j^{\prime} m^{\prime} n^{\prime}}^{j m n}= & \frac{\Phi_{0} \delta_{j^{\prime}}^{j}}{m_{\perp} L}\left(\frac{\pi j}{L}\right)^{2}\left[\delta_{n^{\prime}}^{n}\left(\delta_{m^{\prime}+1}^{m}+\delta_{m^{\prime}-1}^{m}\right)+(m \leftrightarrow n)\right] \\
& +\frac{\Phi_{0}(-1)^{j+j^{\prime}}}{m_{\|} L}\left(\frac{2 \pi}{a}\right)^{2}\left[\frac{j j^{\prime}\left(1-\delta_{j^{\prime}}^{j}\right)}{j^{2}-j^{\prime 2}}-\frac{1}{4} \delta_{j^{\prime}}^{j}\right] \\
& \times\left[\delta_{n^{\prime}}^{n}\left(m+m^{\prime}\right)\left(\delta_{m^{\prime}-1}^{m}-\delta_{m^{\prime}+1}^{m}\right)+(m \leftrightarrow n)\right] .
\end{aligned}
$$

It is clear that there are two very different regimes, $L \gg a$ and $L \ll a$.

The gaps in spectrum can be classified by comparing $j$ and $j^{\prime}$ in the degeneracy points. Degeneracy points with $j=j^{\prime}$, $n=n^{\prime}$, and $m=m^{\prime} \pm 1$ correspond to $q_{y}=\pi(2 m \mp 1) / a$. The corrugation-driven energy gaps in these directions are

$$
\delta E=\frac{2 \Phi_{0}}{m_{\perp} L}\left(\frac{\pi j}{L}\right)^{2}\left|1 \pm \frac{m_{\perp}}{8 m_{\|}}\left(\frac{2 L}{j a}\right)^{2}(2 m \pm 1)\right| .
$$

Expressions for $j=j^{\prime}, m=m^{\prime}$, and $n=n^{\prime} \pm 1$ are similar. For the directions for which $j \neq j^{\prime}, n=n^{\prime}$, and $m=m^{\prime} \pm 1$, the gaps are

$$
\begin{aligned}
\delta E= & \frac{2 \Phi}{m_{\|} L}\left(\frac{2 \pi}{a}\right)^{2} \frac{j j^{\prime}}{\left|j^{2}-j^{\prime 2}\right|} \times\left[\delta_{n n^{\prime}}\left(m+m^{\prime}\right)\right. \\
& \left.\times\left(\delta_{m, m^{\prime}-1}-\delta_{m, m^{\prime}+1}\right)+(m \leftrightarrow n)\right] .
\end{aligned}
$$


The gaps $\delta E$ in the electron spectrum are important when they occur near the Fermi surface, $E_{j m n}^{(0)}=E_{j^{\prime} m^{\prime} n^{\prime}}^{(0)} \sim \epsilon_{F}$, and determine the split between the Fermi curves. Not surprisingly, such special values of the film thickness $L$ include the set of values of $L$ at which one can observe the ETT.

The ETT is described by the distance from the transition, $\lambda_{j}=\left(L_{j}-L\right) / L_{j}$. The formation of a new Fermi loop is the second-order phase transition while the formation or disruption of a neck causes the logarithmic singularities, $\ln \left|\lambda_{j}\right|{ }^{3}$ The singularities are smeared to the extent of $\delta \lambda_{j}$ $\sim m_{\perp}\left(L_{j} / \pi j\right)^{2} / \tau$, where $\tau$ is the relaxation time. Since the film thickness $L$ in reality is a discrete variable, the dependence of observables on the distance from the transition, $\lambda_{j}$, can be measured if the separation of the transition points $L_{j}$, Eq. (4), is larger than the lattice constant $a^{\prime}$. For this, the ratio $m_{\|} / m_{\perp}$ should be small and, preferably, close to the ratio of the bulk and surface periods $a^{\prime} / a$. Otherwise, one will observe just a set of different reconstructions of the Fermi surface for films with different numbers of atomic layers.

The above reconstruction of the Fermi surface and the new gaps on the Fermi surface are meaningful only if $\delta E$ is larger than the scattering-driven line broadening, $\delta E \gg 1 / \tau$, i.e., if the electron mean free path is larger than $a^{\prime} \epsilon_{F} / \delta E$. If the bulk relaxation is caused by the bulk impurities with $1 / \tau \sim n_{i} a^{\prime} \boldsymbol{\epsilon}_{F}$, then $\delta E \gg 1 / \tau$ if

$$
\frac{\Phi_{0}}{m_{\|} L}\left(\frac{2 \pi}{a}\right)^{2}, \frac{\Phi_{0}}{m_{\perp} L}\left(\frac{\pi j}{a}\right)^{2} \gg n_{i}^{1 / 3} \sqrt{\epsilon_{F} / m_{\|}},
$$

where $n_{i}$ is the impurity density. If the relaxation is caused by the scattering by the surface inhomogeneities ${ }^{2}$ with $1 / \tau$ $>8 \pi^{4} l^{2}\left(R^{2} / L a^{\prime 3}\right) \epsilon_{F}$, then $\delta E \gg 1 / \tau$ if

$$
\frac{\Phi_{0}}{m_{\|} L}\left(\frac{2 \pi}{a}\right)^{2}, \frac{\Phi_{0}}{m_{\perp} L}\left(\frac{\pi j}{a}\right)^{2} \gg 8 \pi^{4} \epsilon_{F} \frac{l^{2} R^{2} p_{F}^{3}}{L},
$$

where $l$ and $R$ are the average amplitude and correlation radius (size) of the random surface inhomogeneities. The electron-phonon and electron-electron processes in the ultrathin films are usually less relevant.

As described above, it is technologically possible to prepare films with, e.g., $\Phi_{0} / L \sim 0.1, a / a^{\prime} \sim 5$, and $L / a^{\prime} \sim 30$. For such films, depending on the quantum numbers and $m_{\|} / m_{\perp}$, the new energy gaps at the Fermi surface are quite large, $\delta E / \epsilon_{F} \sim 10^{-4}-10^{-1}$.

The ETT and the gap formation can be observed in the density of states, thermodynamics, and transport experiments. In thin films, the grazing electrons with low quantum numbers $j$ provide a disproportionately large contribution to the conductivity. Thus, near the point $L=L_{1}$, the conductivity $\sigma\left(\lambda_{1}\right)$ exhibits the largest ETT singularity and becomes highly anisotropic because of the band gaps in certain directions. The signs of ETT for higher $j$ in $\sigma(L)$ are much less pronounced. In contrast to this, the transitions at all $L_{j}$ make comparable singular contributions to the density of states or thermodynamic functions.

Experimentally, the singularities in the density of states, and band gaps should be detectable with either a conventional high-resolution photoelectron spectroscopy or scanning tunneling spectroscopy. For the transport property, however, it entails more special capability, such as multiprobe $\mathrm{STM},{ }^{11}$ or specially patented atomically clean nanoelectrodes. ${ }^{12}$

In summary, we predict the existence of a series of ETT in any quantized metal film with periodically modulated surface and the appearance of new band gaps near the edges of the new, modulation-driven, nested Brillouin zone. The ETT's at $L=L_{j}$ [Eq. (4)] lead to the singularities in dependence of the density of states, thermodynamic functions, transport coefficients, etc., on the thickness of the film. The values $L_{j}$, at which the ETT's are observed, are determined almost exclusively by the period of the corrugation and do not depend [if one disregards small shifts of $\left.L_{j}, \delta L_{j}=m_{\perp}\left(L_{j} / \pi j\right)^{2} \delta E\right]$ on the amplitude of the surface corrugation. In contrast to this, the energy gaps $\delta E$ and the amplitude of the singularities are proportional to the amplitude of the corrugation. The energy gaps along certain directions determine the extent of the splits between parts of the Fermi surface at the edge of the Brillouin zone and lead to a strong anisotropy of conductivity near ETT points. These findings could open new ways of tailoring the properties of ultrathin films by means of boundary engineering.

The issue of the controlled reconstruction of the energy spectra in ultrathin films by the periodic modulation of the surface is actually much broader than the issue of ETT. It opens up a new way for tailoring a material's electronic properties simply by controlling its boundary. The periodic modulation of the surface always causes the reconstruction of electron, phonon, and magnon spectra in quantized films and the formation of new energy gaps at the intersections with the boundaries of the new Brillouin zone. These positions are controlled by the period of the modulation and the film thickness. The above calculation method for the width of the gaps can be easily modified for other broadband systems.

One of the authors (A.M.) acknowledges support by the NSF Grant No. DMR-0077266.
${ }^{1}$ I.B. Altfeder, D.M. Chen, and K.A. Matveev, Phys. Rev. Lett. 80, 4895 (1998); I.B. Altfeder, V. Narayanamurti, and D.M. Chen, ibid. 88, 206801 (2002).

${ }^{2}$ A.E. Meyerovich and I.V. Ponomarev, Phys. Rev. B 65, 155413 (2002).

${ }^{3}$ Ya.M. Blanter, M.I. Kaganov, A.V. Pantsulaya, and A.A. Varla- mov, Phys. Rep. 245, 159 (1994).

${ }^{4}$ J.J. Paggel, T. Miller, and T.C. Chang, Science 283, 1709 (1999).

${ }^{5}$ T. Schroeder, J.B. Giorgi, A. Hammoudeh, N. Magg, M. Bumer, and H.-J. Freund, Phys. Rev. B 65, 115411 (2002); E. Vesselli, C. Africh, A. Baraldi, G. Comelli, F. Esch, and R. Rosei, J. Chem. Phys. 114, 4221 (2001). 
${ }^{6}$ A.A. Baski, S.C. Erwin, and L.J. Whitman, Science 269, 1556 (1995).

${ }^{7}$ A. Kirakosian, R. Bennewitz, J.N. Crain, Th. Fauster, J.-L. Lin, D.Y. Petrovykh, and F.J. Himpsel, Appl. Phys. Lett. 79, 1608 (2001).

${ }^{8}$ S. Tanaka, C.C. Umbach, J.M. Blakely, R.M. Tromp, and M. Mankos, J. Vac. Sci. Technol. A 15, 1345 (1997); H.H. Solak, D. He, W. Li, S. Singh-Gasson, F. Cerrina, B.H. Sohn, X.M. Yang, and P. Nealey, Appl. Phys. Lett. 75, 2328 (1999).

${ }^{9}$ Z. Tesanovic, M.V. Jaric, and S. Maekawa, Phys. Rev. Lett. 57,
2760 (1986); N. Trivedi and N.W. Ashcroft, Phys. Rev. B 38, 12 298 (1988); A.E. Meyerovich and S. Stepaniants, Phys. Rev. Lett. 73, 316 (1994).

${ }^{10}$ A.E. Meyerovich and B.E. Meyerovich, Zh. Eksp. Teor. Fiz. 93, 1461 (1987) [Sov. Phys. JETP 66, 833 (1987)].

${ }^{11}$ M. Aono, APS Bull. 47, 97 (2002); S. Hasegawa, I. Shiraki, and F. Tanabe, ibid. 47, 98 (2002).

${ }^{12}$ U. Ramsperger, T. Uchihashi, and H. Nejoh, Appl. Phys. Lett. 78, 85 (2001). 